\title{
Analysis of Psychological Spouse Abuse against Men in Iranian Couples: A Qualitative Study
}

\author{
Ahmad Nouri ${ }^{1}$, Ozra Etemadi ${ }^{1}$, Rezvanossadat Jazayeri ${ }^{1}$ \& Maryam Fatehizade $^{1}$ \\ ${ }^{1}$ Department of Counseling, Faculty of Education and Psychology, University of Isfahan, Isfahan, Iran. E-mail: \\ O.etemadi@edu.ui.ac.ir
}

Correspondence: Ozra Etemadi, Department of Counseling, Faculty of Education and Psychology, University of Isfahan, Isfahan, Iran. Tel: 98-313-793-2515. E-mail: O.etemadi@edu.ui.ac.ir

Received: March 13, 2016

Accepted: April 11, 2016

Online Published: June 6, 2016

doi:10.5539/res.v8n3p1

URL: http://dx.doi.org/10.5539/res.v8n3p1

\begin{abstract}
This study aimed to explore the psychological spouse abuse against men in a sample of Iranian couples. This qualitative study used the grounded theory in triangulation. The statistical population consisted of a compilation of electronic resources, books, theses, and journals, family counseling experts' opinions and men who suffered from spouse abuse in Isfahan. Purposeful sampling began and continued until saturation of the categories. Data collection consisted of 15 semi-structured interviews with abused married men, 10 interviews with family counseling experts and content analysis of books, articles, and journals related to psychological spouse abuse. The results showed that some cases of spouse abuse against the men are neglecting the spouse' needs, lack of commitment and accountability, arbitrary behavior, turning to anti-moral values and some habits and annoying personality traits. Spouse abuse is rooted in social, religious, economic, environmental, cultural backgrounds and family life. Intervening factors include factors related to the others, personality traits of the abused person and background factors. The causal factors include the interpersonal conditions and personality types. The most important strategy for men against spouse abuse include aggressive-confronting response, silence, peaceful, defense, reform reaction and alternative reaction. The psychological consequences of marital violence include, individual, interpersonal, family, and social consequences. As a result, psychological spouse abuse against men is affected by a number of factors that were discussed in this study.
\end{abstract}

Keywords: psychological spouse abuse, spouse abuse, qualitative study

\section{Introduction}

Marital relationship is the most important and most fundamental human relationship since it provides a basic structure for family relationships and raising the future generation (Larson \& Holman, 1994). World Health Organization, introduced family as the social core of increasing health and wellbeing (Campbell, 2003). Various studies also suggest that happy mutual life help people live happier, healthier (Dannelly, 1993). Nevertheless, if the marital relationships are distorted, it leads to the destructive and negative effects on the mental health of family and children. Several studies have confirmed that marital problems have negative consequences for mental and physical health in mutual life and cause the progressive diseases such as changes in immune system, cardiovascular diseases and malfunction of the endocrine system (Whitson \& El-Sheikh, 2003). Marital quality and the factors, which affect it, should be considered due to their effect on the psychological health of the couples and children. Experts believe that identification and treatment of harmful factors on the quality of the mutual life can be undeniable on maintaining and promoting the mental health of the couples (Byrne et al., 2004). Nowadays, spouse abuse is one of the main problems of public health with short and long-term consequences on physical and mental health of the couples (Campel, 2002).

Marital conflict is caused by a reaction to the individual differences and sometimes it is so severe that leads to the feeling of anger, abuse resentment, hatred, jealousy, and physical and verbal misbehaviors in couple's relationships in the form of destructive scenarios. Barnett (2012) defines violence as a pattern of abusive behavior against another person with the aim to implement and maintain power and control over another person. Spouse abuse in its broadest sense refers to the misbehaviors of one of the spouse towards another. A number of scholars define spouse abuse as using the ways to force the other to do or not to do something despite his or her propensity (Jazayeri \& Sharifi, 2014). 
Ganly (1981) sorts four types of family violence: a) emotional violence, b) physical violence, c) verbal violence, and d) sexual violence. Emotional violence is a process in which an individual is psychologically injured by someone else, little by little. Ronaldmyro (2001) identifies four dimensions of emotional violence: disgracing behavior that corrupts the self-esteem of the spouse including yelling, discrediting and criticizing the husband. The second dimension is passive violence; withholding the social protection of the husband. The third dimension includes changing and the implying the threatening behaviors. The fourth dimension is to limit the scope of the person and freedom of the spouse. In another study, violence is divided into four categories: physical, psychological, sexual and emotional (Jazayeri et al., 2014). This study focuses on analyzing the causes of psychological spouse abuse. Behaviors such as cursing, blaming, taunting, threatening, slandering, or anything that disturbs the mental peace of the spouse are regarded as the psychological violence (Jazayeri et al., 2014).

Violence is a risk factor and an obstacle to the marital happiness. Studies that focus on the relationship between spouse abuse and divorce support the idea that spouse abuse more likely leads to the divorce (Arias et al., 1997; Katz et al., 1995). Sudha and Morrison (2011) showed that spouse abuse might have a negative impact on multiple aspects of the women's reproductive health, including the increase in self-report of symptoms of sexually transmitted diseases. Unfortunately, gender has not been much considered in a high number of studies. At the beginning, violence in intimate relationship was attributed only to the men because data were derived from the reports of the police that were usually related to the severe violence. However, new studies have shown that women can commit violence (Mohammad Khani et al., 2010).

In conducting the studies, men and women are not investigated separately and only the men's violence against the women has been studied (Heaton \& Blake, 1999). A number of studies show the similar rate of violence and even some have concluded that violence is more prevalent among the women than men (Archer, 2002). Abusive and Environments (2010) using an analysis of 302 men abused by their wives, showed that 35 percent of men have experienced severe physical damage and $23 \%$ of them suffer from mental illness, such as depression or anxiety. Theories of spouse abuse have been discussed as follow. According to social learning theory, Bandura (1986) focuses on the process of learning to be angry, through observation and imitation. From this perspective, behaviors are taught through imitation (imitating the behavior of family members or others) and through direct experience. If people can satisfy their needs by this behavior, it more likely continues even with no reward notably if this behavior is offered by a person who is respected for the observer. Straus et al. (1980) regards that people who like each other, show violence too. Lack of moral limitations on violence and permitted violence are the causes of violence when there is no other solution.

According to functionalism theory, this theory justifies anger. They consider the family as a group where there is a long relationship between people with different genders and age groups that in spite of other groups, hierarchy is not based on expertise, competence, or merit. But, it is based on gender, age, economic power, and the existence of the conflicting interests that this lead to the explicit or implicit conflicts in this heterogeneous group. In this theory, resolving the family conflicts by violence is possible. Violence plays a role in the natural flow of daily life; family members focus on their basic functions. Women responsibilities include family duties and main the men as the breadwinner is communicating with the outside world (Coker, 1978).

According to the exchange theory, people seek to maximize your profits and minimize their costs in their interpersonal relationships. As a result, only if the people involved in a relationship enjoy the balanced cost-profit, they continue their relationships. From this perspective, in a violent marital relationship, the cost-profit ratio is as follows: the husband beats his wife and from his perspective, this behavior is right because when woman disobeys, there is no balanced cost-profit relationship and he prefers to end this situation. He supposes that a balanced situation is provided using a violent behavior, and when he achieves it, he feels self-confident (Goode, 1971; Wallace \& Roberson, 2015).

Feminist theory avoids using the broad concepts such as family violence or husband abuse and so on and claims that men frequently show violence against their wives and children and the main reason for violence is the authoritarian patriarchal structures in society. The family, as the central patriarchal foundation, is based on social inequality and exploitation of women and children and ideologists seek to maintain it. In their view, male violence reflects a system of sovereignty of all men on all women that has been observed in the historical and comparative studies (Dekeseredy \& Halle, 1993). Of course, this view is used on men's violence against women. It does not believe that men may be suffered from women's violence.

The results indicate that the rate of spouse abuse in the family is one of the common problems. Therefore, identifying the factors influencing the spouse abuse in the family is important. Given that in previous studies and 
different theories, spouse abuse has been divided into different types, this study aims to analyze the psychological spouse abuse against the men.

\section{Method}

This qualitative study used the grounded theory in triangulation for increasing the validity of the study. The statistical population consisted of a compilation of electronic resources, books, theses, and journals related to psychological spouse abuse, family counseling experts' opinions and men who suffered from spouse abuse in Isfahan.

For determining the predictors of psychological spouse abuse in the literature, 29 references (Latin: 1980-2014 and Persian: 2001-2015) were selected to the saturation of the categories and analyzed. Furthermore, 15 abused men among the qualified men were selected using the purposive sampling method and with maximum variance of sampling (in terms of age, duration of mutual life, different stages of family life cycle, economic and social levels, educational level) to the saturation of the categories. Inclusion criteria are as follow:

1) Identification of the psychological spouse abuse in men using a preliminary interview.

2) Failure to identify the personality disorders or severe clinical disorders that were analyzed using the introductory interview by the researcher and semi-structured diagnostic interview, if necessary.

3) Lack of substance abuse and alcohol.

4) Interest in and willingness to participate voluntarily in the research and expressing the experiences.

Ten experts with at least 3 years of counseling experience with couples who were interested to participate in an interview were selected from among the family counseling experts using the purposive sampling method.

\subsection{Research Tools}

Semi-structured interview: This interview was conducted using qualitative approach from the broad and general questions to the exploratory questions about the psychological violence to obtain precise information about the spouse abuse and family-counseling experts. Interview time was 40-120 minutes based on the conditions and participants' tendencies.

All interviews were digitally recorded was immediately transcribed after the recording. To increase the reliability and validity-that show the truth wording of the findings in a qualitative study-prolonged engagement of the researcher, contacting and communicating with participants to obtain their trust and understand the experiences were considered by the researcher.

Maximum diversity of participants increased the data reliability. In addition, the methods of data credibility were used by checking the manuscripts by the members in order to remove any coding ambiguity. For this purpose, the researcher provided them with some parts of their interviews and coding to achieve similar concepts in relation to statements made by the participants. The data saturation was used to increase the reliability of the study.

Data were analyzed using a systematic method and simultaneously with the data collection. In this way, the qualitative data obtained from interviews and other documents were analyzed manually and using coding in three stages, open, axial, and selective. In open encoding, researcher reviewed data line by line and derived its core concepts. Two coding methods were used in this stage: language and words of the interviewee and codes and key concepts that researcher derived based on the existing concepts in data. Then, using the axial coding, the extracted concepts were classified and the isolated raw concepts that were apparently unrelated but interdependent were brought together.

In this way, the initial codes were decreased to the categories and the main axes were identified in the data set. Then, each category was compared to other category in order to ensure the fact that categories are separated. For identifying the categories, the relationship between the categories, subcategories and main categories was explored and the dimensions and features of each category were arranged. In the third stage or selective coding, final selection was made using the concepts and identified codes, and the researcher aimed to integrate and refine his analyses (Bazargan, 2010). 


\section{Findings}

At first, results of the interviews and text analysis were openly and then, axially coded. The core issues in psychological spouse abuse against the men were explored using the conceptual precise classification and accurate proportions.

Data analysis showed that cases of spouse abuse against men include neglecting the husbands' needs, lack of commitment and accountability, arbitrary behavior, turning to anti-moral values and some habits and annoying personality traits. Spouse abuse is rooted in social, religious, economic, environmental, cultural backgrounds and family life. Intervening factors include others' role, personality traits of the abused person and background factors, and the causal factors include the interpersonal conditions and personality types. The most important strategy for men against spouse abuse include aggressive-confronting response, silence, peaceful reaction, defense reaction, reform reaction and alternative reaction. The individual, interpersonal, family, and social consequences of spouse abuse, among others, are of great importance. As a result, psychological spouse abuse against men is affected by a number of factors that were discussed in this study. Axial extracted categories include neglecting the husbands' needs, arbitrary behavior, turning to anti-moral values and some habits and annoying personality traits. The extracted model obtained from data analysis is shown in Figure 1.

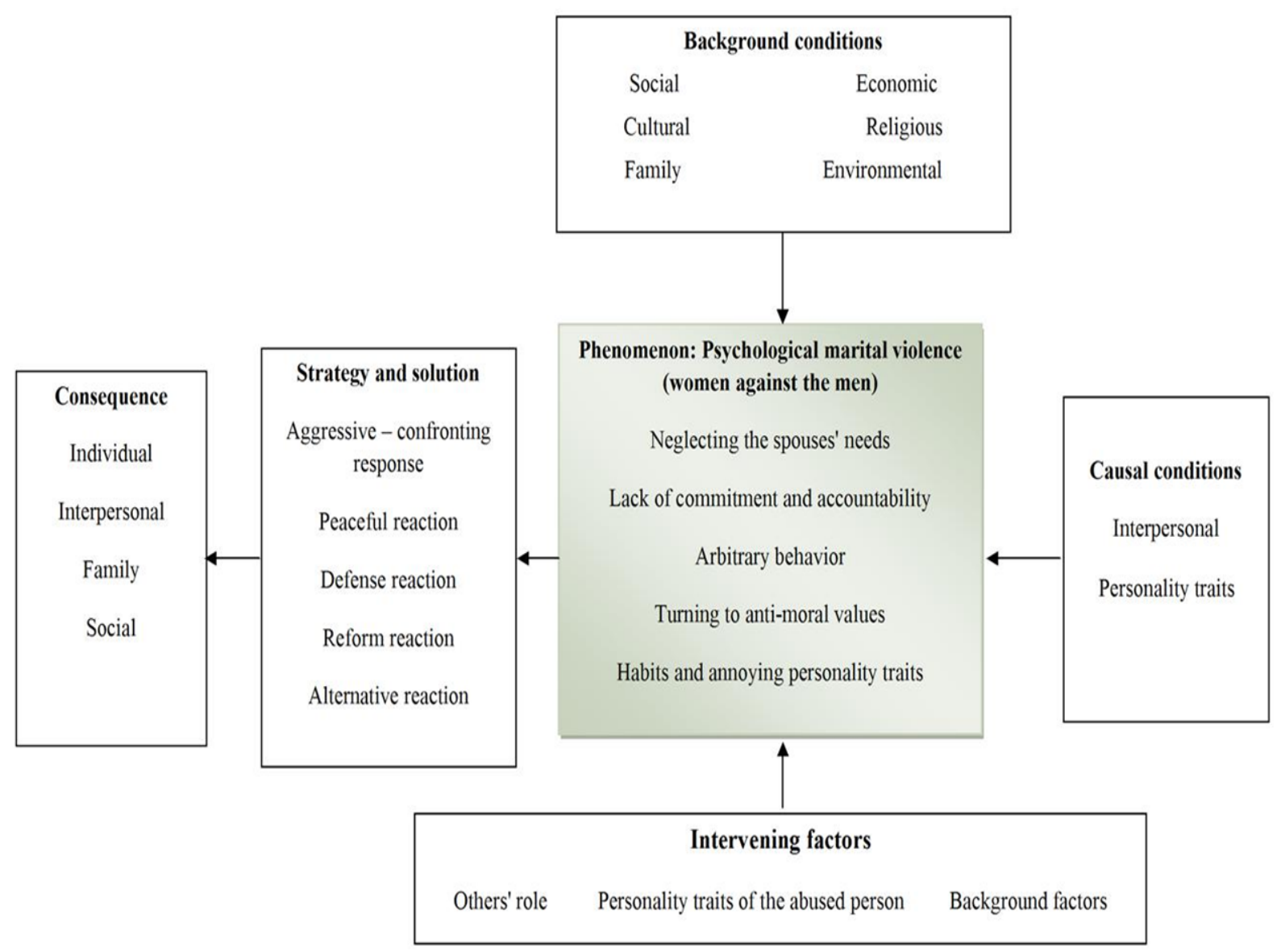

Figure 1. Model of psychological spouse abuse against the men

\subsection{Conceptual Manifestations of Psychological Husband Abuse against the Men}

1) Neglecting the husband in the form of:

A) Neglecting the communication and interaction with husband's family

- Rigidity and formal relationships with the husbands family

- Financial and economic independence of husband's family 
- Separation of the living place from husband's family

- Neglecting and disrespecting for the husband's family

B) Neglecting and emotional coldness towards the husband

- Colluding with children against the men and abandoning the husband

- Too much attention to the financial and intellectual issues of their families

- Spending too much with friends and not allocating the time for the husband

- Cold greeting the husband when he comes home

- Neglecting the husband's efforts to meet the needs of his family

- Neglecting the husband and paying attention to other things

- Too much entertaining with the virtual environments

- Hostile and deliberate abdication of the husband

- Non- intimate relationships with the husband

2) lack of commitment and accountability in the form of women's irresponsibility and lack of commitment to their husbands and children as a sign of violence

- Discouragement and apathy to do daily chores

- Neglecting the daily chores

- Evading the tasks such as raising the children and cleaning the house

- Not cooking

- Teasing the children

3) arbitrary behavior in the form of:

A) communication and social arbitrariness

- Checking the calls and messages on the mobile and controlling his commutations

- Showing sensitivity in husband's communications with others

- Mistrusting and controlling

- Limiting the men's interests

- Limiting the husband's contracts with his friends

- Limiting the husband's contracts with his family

- Limiting the common contracts with others

- Limiting natural freedom

- Limiting the social interactions, such as separating the husband from his family

B) expectations and arbitrary thoughts and behaviors

- An exceptional financial situation in the shortest possible time

- The fulfillment of all needs in any way

- The man should never feel tired and always should show respect and affection

- The man should provide his wife all things she needs; expecting the womanly behaviors of the husband as an illogical expectation

- Illogical financial expectation

- Permanent admiration by the husband

- Ordering the husband because of having a better financial situation

- Sense of leadership and management

- Controlling the husband

- Attempts to correct the husband's personality traits according to her desire

- Ambitious and arbitrary nature of the woman in relation to his husband in front of others 
4) turning to anti-moral values in the form of:

- Selfishness

- Despising, ridiculing and insulting

- Humiliating the husband in front of others

- Blaming and rebuking

- Comparing

- Reproaching

- Finding faults

- Pride and megalomania

- Narcissism

- Slandering

- Sarcastic talking

- Long the debates and discussions

- Picking on the husband

5) habits and annoying personality traits in the form of:

- Manly behaviors

- Lavishness

- Nnagging and making vain excuses

- Victimization in front of others and exaggerating the mistakes of the husband

- Prejudice and mind reading

- Anxiety and stress

- Obsession

- Impressedness and irresoluteness

- Rashness

- Ambitiousness

- Rivalry

- Superstitious behaviors

- Irritability

- Jealousness

- Lack of social interaction

- Feigning illness

- Stingy

3.2 Background Factors of the Psychological Spouse Abuse

(1) Social context (social issues, the negative role of the media, the consent of the people)

(2) Religious background (poor religious beliefs, religious prejudice)

(3) Economic (financial problems)

(4) Family background (education among the boys and having the manly behaviors, spoiled behaviors, poor financial situation of the paternal family, culture and family relationships, illiteracy and superstitious of the woman's family, the impressedness of parental misconduct in childhood)

(5) Environmental (ecological and climatic conditions, characteristics living place)

(6) Cultural (poor-informed or mediatory marriage, emphasis on early marriage and lack of intellectual, psychological and economic maturity to getting married, traditional ways of choosing the husband, low educational level of women and men) 


\subsection{Intervening Factors}

A) Factors related to the others (secret relationship with another person outside the mutual life, having children and the role conflict of the parent-husband, living with the main family).

B) Characteristics of the abused person (women's physical and mental disease, working women, addiction and abuse of alcohol and drugs).

C) Background factors underlie (his poor economic status of the man, extreme respect for the woman, behavioral faults of men) including examples of the intervening factors in psychological spouse abuse against men.

\subsection{Causal Factors}

A) Interpersonal conditions (lack of compatibility, emotional marriages, lack of realism in marriage and hope to change their husband's behavior, lack of counseling before marriage with others, lack of familiarity with the techniques that show how they should treat with their husbands, lack of attention to the families' opinions when getting married, feeling of being oppressed and lack of satisfaction of needs, love addiction)

B) Personality types that are divided into two general personality types: Abuser (personality traits) and abusive (personality traits)

\subsection{The Most Important Strategy for Men against the Spouse Abuse}

A) Aggressive-confronting reaction (angriness and beating, swearing, expressing aversion, do things contrary to the husband's desires, indifference to the angry husband, threatening, teasing)

B) The reaction of silence including being silent and lack of attention towards annoying behavior of the husband

C) Peaceful reaction (going out after an argument, smiling to attract the husband' affection, positive silence in order to delay the response, silence at the moment of angriness)

D) Defensive reaction (controlling anger and patience, silence, concessions and avoiding the sensitive behaviors)

E) corrective reactions (talking and trying to change behavior, modifying their imperfections, asking help from others, using the resources and improving information)

F) Alternative reaction shows the entertaining with the different things that the abused person avoids them.

Consequences of psychological spouse abuse

A) Individual outcome (impaired daily functioning, brain disorders, psychosomatic disorders, and sexual disorders, tendency to abuse drugs and alcohol, a sense of guilty, psychological injury in the annoying person, psychiatric disorders, and physical disorders)

B) Interpersonal (emotional divorce, legal divorce, infidelity)

C) Family (academic drop of the children, the adverse effect on children's physical and mental health, negative modeling based on the misbehaviors of the parents, creating hatred in children, reducing the positive energy of the family members)

D) Social (deficiency in communications and social interactions with family, friends and others, failure to perform duties and social functions, change the abused person to the annoying person in future, disrupting the healthy social relationships)

\section{Discussion and Conclusion}

This study aimed to explore the psychological spouse abuse against men. Data analysis showed that cases of spouse abuse against men included neglecting the husbands' needs, lack of commitment and accountability, arbitrary behavior, turning to anti-moral values and some habits and annoying personality traits. Moreover, background, causal, and intervening factors were investigated. Eventually, the abused person's reactions against the psychological violence and consequences of spouse abuse were discussed and a number of cases were introduced. As a result, although the cases of psychological spouse abuse against the men were psychologically discussed the background factors, the causal factors and intervening factors may not be interpreted in the same way. Consequently, psychological spouse abuse is influenced by different factors.

Results showed that violence is mainly related to culture. Violence, by its very nature, is not defined as all behaviors that lead to others' mental or physical discomfort or injury. Perhaps, somebody suffers mentally or even physically from a correct, logical, and rational behavior that is common in a certain culture; For example, a sense of personal grandiosity leads to having the false expectations and considering the others' normal behaviors as offensive and insulting. 
In the functionalism approach, conflicts within the family are responded with the anger. This study showed that spouses might lose temper in response to conflicts within the family. According to the exchange approach, aggressive reciprocal behavior of the men is a coping reaction against the ventilation. Here, women think that they have been oppressed, therefore, try to compensate and since they are emotional, they appeal to the emotional abuse. Contrary to the feminist theory that claims only men are violent against the women, this study showed that women-for some reasons-are also violent against the men, but in a different way.

It means that some behaviors are commonly and rationally offends others. Thus, there are slightly different cases of violence in different cultures, and there is no certain stereotype for violence in all cultures. For example, in societies where the men are considered legally and culturally as the financial supporter of the women, the lack of economic support could be a case of violence. However, in societies where the men are not responsible to do this, the lack of financial support does not lead to the violence. Alternatively, in some cultures, women should care of their husband's ill parents and this is culturally common and is not a case of violence, however, if the women disobey, it is considered as her fault. Moreover, in some cultures, women should not do daily chores; however, in some others, it is common and even exhilarating to do that.

Previous studies investigated the causes of spouse abuse against women and this issue has been less considered from a qualitative viewpoint. However, this study explored psychological spouse abuse against men based on the grounded theory. Therefore, the present study, compared to the previous studies, is of great preference and value. Interviewees and their investigation, analysis of limited resources, concentration on the psychological spouse abuse and ignoring other dimension are of the limitations of this study that should be taken into account I future studies. Finally, it is suggested to develop treatment programs on spouse abuse based on the components of this study and to examine the cases of spouse abuse in different cultures.

\section{References}

Abusive, S., \& Environments, V. (2010). Domestic violence programs discriminate against male victims. Rockville, $M D$.

Archer, J. (2002). Sex differences in physically aggressive acts between heterosexual partners: A meta-analytic review. Aggression and violent behavior, 7(4), 313-351. http://dx.doi.org/10.1016/S1359-1789(01)00061-1

Arias, I., Lyons, C. M., \& Street, A. E. (1997). Individual and marital consequences of victimization: Moderating effects of relationship efficacy and spouse support. Journal of Family Violence, 12(2), 193-210. http://dx.doi.org/10.1023/A:1022888728475

Bandura, A. (1986). Social foundations of thought and action: A social cognitive theory. Prentice-Hall, Inc.

Barnett, A. (2012). Effects of Domestic Violence on the Workplace.

Byrne, M., Carr, A., \& Clark, A. (2004). The efficacy of behavioral couples therapy and emotional focoused therapy for couple distress. Contemporary family therapy, 26(4), 361-384. http://dx.doi.org/10.1007/s10591-004-0642-9

Campbell, T. L. (2003). The effectiveness of family interventions for physical disorders. Journal of Marital and Family, 29(2), 263-281. http://dx.doi.org/10.1111/j.1752-0606.2003.tb01204.x

Coker, J. B. (1978). Violence and the Family. In J. P. Martin, \& J. P. Martin (Eds.). Wiley.

Dannelly, D. A. (1993). Sexually inactive marriages. Journal of Sex Research, 30(2), 171-176. http://dx.doi.org/10.1080/00224499309551698

Dekeseredy, W. S., \& Kelly, K. D. (1993). The incidence and prevalence of woman abuse in Canadian university and college dating relationships. Canadian Journal of Sociology, 18, 137-159. http://dx.doi.org/10.2307/3341255

Goode, W. J. (1971). Force and violence in the family. Journal of Marriage and the Family, 624-636. http://dx.doi.org/10.2307/349435

Heaton, T. B., \& Blake, A. M. (1999). Gender differences in determinants of marital disruption. Journal of Family Issues, 20(1), 25-45. http://dx.doi.org/10.1177/019251399020001002

Jazayeri, R., Etemadi, O., Mortazavi, M., \& Fatehizade, M. (2014). Marital violence theory and treatment. Golbang publications, Yazd.

Katz, J., Arias, I., Beach, S. R., Brody, G., \& Roman, P. (1995). Excuses, excuses: Accounting for the effects of partner violence on marital satisfaction and stability. Violence and Victims, 10(4), 315-326. 
Mohammadkhani, P., Azadmehr, H., \& Motaghi, S. (2010). Association between the Types of Violence in Couple Relationship and Marital Attitudes in Sexual Coerced Victims. Iranian Journal of Psychiatry and Clinical Psychology, 16(3), 195-202.

Straus, M. M. A., Gelles, R. J., \& Steinmetz, S. K. (Eds.). (1980). Behind closed doors: Violence in the American family. Transaction Publishers.

Sudha, S., \& Morrison, S. (2011). Marital violence and women's reproductive health care in Uttar Pradesh, India. Women's health issues, 21(3), 214-221. http://dx.doi.org/10.1016/j.whi.2011.01.004

\section{Copyrights}

Copyright for this article is retained by the author(s), with first publication rights granted to the journal.

This is an open-access article distributed under the terms and conditions of the Creative Commons Attribution license (http://creativecommons.org/licenses/by/3.0/). 